Meta

Journal des traducteurs

Translators' Journal

\title{
La formation du traducteur arabe : le cas de la mise à niveau linguistique
}

\section{Jarjoura Hardane}

Volume 45, numéro 3, septembre 2000

La traduction dans le monde arabe

URI : https://id.erudit.org/iderudit/003025ar

DOI : https://doi.org/10.7202/003025ar

Aller au sommaire du numéro

Éditeur(s)

Les Presses de l'Université de Montréal

ISSN

0026-0452 (imprimé)

1492-1421 (numérique)

Découvrir la revue

Citer cet article

Hardane, J. (2000). La formation du traducteur arabe : le cas de la mise à niveau linguistique. Meta, 45(3), 475-479. https://doi.org/10.7202/003025ar
Résumé de l'article

Le degré de maîtrise des langues seconde et troisième chez les étudiants arabes n'est pas l'unique obstacle que ceux-ci affrontent lors de leur formation en traduction ou en interprétation: l'arabe classique est également une difficulté de taille. Pour la surmonter, les étudiants devraient prendre, dans les trois langues, des cours qui leur permettraient de structurer leurs habiletés cognitives et d'améliorer leur langue. On admet que, tout au long de sa carrière, le traducteur doit absolument travailler à atteindre une excellente compétence linguistique. 


\title{
La formation du traducteur arabe: le cas de la mise à niveau linguistique
}

\author{
JARJOURA HARDANE \\ École de traducteurs et d'interprètes de Beyrouth, \\ Université Saint-Joseph, Liban
}

\begin{abstract}
RÉSUMÉ
Le degré de maîtrise des langues seconde et troisième chez les étudiants arabes n'est pas l'unique obstacle que ceux-ci affrontent lors de leur formation en traduction ou en interprétation: l'arabe classique est également une difficulté de taille. Pour la surmonter, les étudiants devraient prendre, dans les trois langues, des cours qui leur permettraient de structurer leurs habiletés cognitives et d'améliorer leur langue. On admet que, tout au long de sa carrière, le traducteur doit absolument travailler à atteindre une excellente compétence linguistique.
\end{abstract}

\begin{abstract}
The level of Arab students in second and third languages is not the only obstacle students face when being trained to translate and/or interpret; Classical Arabic is also a major hindrance. In order for students to overcome this handicap, they should follow courses in the three languages - that would enable them to communicate in real settings, to organize their cognitive skills, and also to improve their language. The pursuit of high linguistic competence is considered imperative for a translator throughout his career.

MOTS-CLÉS / KEYWORDS

langues seconde et troisième, compétence communicative, mise à niveau linguistique
\end{abstract}

\section{Introduction}

Les programmes de la plupart des écoles de traduction et d'interprétation ne prévoient pas de mise à niveau dans la première langue choisie par l'étudiant. Par contre, la mise à niveau dans les deux autres langues de la combinaison linguistique, le plus souvent proposé sous la forme d'un séjour à l'étranger, y occupe une place importante. La formation des traducteurs et des interprètes arabes ${ }^{1}$ nous permet de faire une série d'observations. D'abord, la mise à niveau en arabe, choisi comme première langue, est incontournable. Ensuite, les séjours linguistiques à l'étranger ne constituent pas l'unique solution pour une mise à niveau en deuxième et troisième langues. Par ailleurs, la mise à niveau linguistique suppose une autre mise à niveau relative à la structuration de la pensée. Enfin, cette double mise à niveau, s'étalant jusqu'à une date récente sur plus d'une année universitaire, est de plus en plus proposée en cours intensifs au début du cycle de formation commun aux candidats à la traduction et à l'interprétation.

\section{Mise à niveau en arabe}

Il serait surprenant, voire contradictoire, de proposer à un étudiant en traduction et en interprétation une mise à niveau dans sa première langue. Mais pour un étudiant 
arabophone, l'arabe littéraire, choisi comme première langue, ne constitue pas le moyen le plus spontané de la communication quotidienne, comme le serait une véritable langue maternelle. En effet, l'arabe littéraire est surtout utilisé dans les «situations de communication écrite» (Martinet 1967: 159) : dans la presse, dans l'édition, dans la correspondance officielle ou privée... Mais lorsque les Arabes «parlent, chantent, murmurent leurs prières, cajolent leurs bébés, chuchotent à l'oreille de ceux qu'ils aiment [...] ou injurient ceux qu'il leur plaît d'injurier, c'est avec le dialecte qu'ils le font» (Frayha 1980). Leur véritable langue maternelle, c'est évidemment le dialecte. Et s'il est vrai que le dialecte et le littéraire peuvent être considérés comme deux états d'une même langue, il n'en reste pas moins que le futur traducteur ou interprète n'utilisera dans l'exercice de son métier que l'arabe littéraire.

Or, l'étudiant arabophone qui arrive au seuil de l'université, après douze années d'études à raison de six heures d'arabe en moyenne par semaine, est loin d'avoir acquis une compétence en arabe littéraire lui permettant d'entreprendre des études de traduction ou d'interprétation. Cette carence se manifeste essentiellement au niveau de la communication orale. Elle est due à la priorité accordée à l'écrit dans les cours d'arabe couvrant les différents cycles scolaires. Le résultat est que les activités d'expression orale sont plutôt rares et que, pendant ces activités, les élèves et même les enseignants glissent naturellement et sans aucune mauvaise conscience vers le dialecte et ne développent ainsi aucune compétence dans l'expression orale en arabe littéraire.

Mais il serait faux de croire que cet étudiant a acquis une compétence satisfaisante dans la communication écrite. Il se montre incapable en fin de parcours d'écrire une petite lettre, de faire le résumé d'un texte ou de rédiger la fiche de lecture d'un livre. Cette carence est la conséquence des pratiques pédagogiques qui s'appuient dans les différents cycles sur «la mémorisation et laissent peu de place à l'expression personnelle» (Hobeika 1997-1998: 58). Elle est aussi le résultat des programmes et des matériels pédagogiques limités au seul registre de la littérature.

\section{Mise à niveau en deuxième et troisième langues}

La compétence de communication orale et écrite de ce même étudiant ne se révèle pas meilleure dans la deuxième ou la troisième langue, malgré le fait que dans les pays arabes bilingues, voire trilingues, l'enseignement de la deuxième langue est introduit en même temps que l'arabe et que celui de la troisième langue commence au plus tard en première année du cycle complémentaire. Les défaillances communicatives proviennent là aussi des pratiques pédagogiques qui considèrent la langue comme un objet d'observation et de description et non comme un véritable «instrument de communication» (Martinet 1967: 20).

Pour la mise à niveau en deuxième et troisième langues, l'idéal reste, aux yeux de plusieurs écoles de traduction, l'organisation de séjours linguistiques à l'étranger. Ainsi est-il généralement admis que cette méthode "naturelle» proposant un «authentique bain linguistique» (Besse 1985: 24) offre les meilleurs moyens pour l'apprentissage ou le perfectionnement d'une langue. En effet, pour apprendre et perfectionner une langue, il ne suffit pas de s'enfermer dans un bureau ou dans une classe, il faut s'ouvrir «pour la capter à la source» (Dejean Le Féal 1976: 51).

Mais ce serait une erreur de «croire qu'il suffit de vivre à l'étranger pour apprendre à la perfection la langue de ce pays » (Dejean Le Féal 1976: 41). En effet, le succès 
de la méthode «naturelle» suppose, en dehors de l'organisation du séjour, plusieurs autres conditions: programmation des activités, disponibilité d'un bon professeur pour le suivi des travaux individuels et pour l'acquisition de l'auto-correction. Et quoi qu'il en soit, elle reste très coûteuse et n'est pas à la portée de tous.

Pour pallier la difficulté d'organiser des séjours à l'étranger, les étudiants arabophones suivent dans leur école des cours de mise à niveau proposés dans les trois langues de leur combinaison linguistique. Et c'est ainsi que plusieurs d'entre eux sont systématiquement sauvés. Mais si le sauvetage est possible, c'est surtout grâce à la manière dont les cours de mise à niveau sont conçus et appliqués.

\section{Les cours de mise à niveau, une problématique en perpétuelle évolution}

Les cours de mise à niveau doivent au point de départ respecter les deux principes élémentaires de toute opération pédagogique susceptible de réussir. Le premier consiste à amener les étudiants vers l'objectif fixé en fonction de leurs besoins réels. Le second principe consiste à considérer que tous les étudiants sont capables d'atteindre cet objectif, mais que chacun l'atteint à sa manière. Les capacités d'apprentissage n'obéissent pas aux critères de l'infériorité ou de la supériorité mais à celui de la différence. Les étudiants diffèrent par «leur profil» (De La Garanderie 1980). Ils diffèrent aussi par leur vécu personnel, l'environnement socio-culturel qui les a façonnés et les moyens pédagogiques qui ont été mis à leur disposition. Le tout est de les aider à découvrir leur profil et à prendre conscience de leurs aptitudes.

Mais si le respect de ces deux principes est nécessaire au sauvetage par les cours de mise à niveau, il ne saurait lui être suffisant. Aussi faut-il déterminer clairement l'objectif de ces cours et choisir les supports et la stratégie qui leur sont adéquats.

L'objectif des cours de mise à niveau est d'assurer aux étudiants la maîtrise d'une compétence communicative opérationnelle à l'oral et à l'écrit. Il est vrai que l'oral intéresse plus l'étudiant en interprétation que l'étudiant en traduction et que le dernier s'attelle plus à l'écrit qu'à l'oral. Mais au début d'un cycle de trois ans commun à la traduction et à l'interprétation, un cours de mise à niveau ne peut adopter qu'une approche globale incluant de façon dynamique les activités propres à l'oral et celles spécifiques de l'écrit.

Mais cette compétence communicative ne se réduit pas à l'utilisation correcte, voire parfaite de la langue. Elle suppose une bonne "structuration de la pensée» (Kfouri Khoury 1998: 7-10) et une bonne organisation dans la méthodologie du travail. Savoir prendre des notes, dégager ou établir un plan, faire un résumé ou écrire un rapport ne relève pas uniquement des seuls acquis linguistiques. Ce sont des démarches que seule une «tête bien faite» (Buzan 1984) peut effectuer avec succès.

Cependant, il serait illusoire d'entraîner les étudiants à acquérir et maîtriser ces démarches sans aucun souci de la compréhension et de l'expression purement linguistiques. C'est ce qu'observent de plus en plus les enseignants et les étudiants utilisant parallèlement des matériels de mise à niveau linguistique et des matériels de ce qu'il est convenu d'appeler " les techniques de travail et d'expression ». Un cours opérationnel de mise à niveau ne saurait dissocier ces deux volets (Awaiss 19971998 : 3-4) de la communication et devrait intégrer les activités visant à la structuration de la pensée et celles entraînant à la compréhension et à l'expression.

Un pareil cours peut paraître excessivement centré sur l'aspect communicatif de 
la langue, en mettant de côté la connaissance approfondie de la grammaire que certains considèrent comme une condition nécessaire et préalable à la mise à niveau. Mais, tout en soulignant l'importance de l'auto-correction grammaticale pour un traducteur ou un interprète et la nécessité de prévoir des séances de grammaire dans les cours de mise à niveau, nous estimons que le génie de la langue est autre chose que l'apprentissage de sa grammaire et que, globalement, le premier peut se passer du second. «C'est ainsi que les experts dans les règles de grammaire peuvent se montrer incapables de s'exprimer correctement, même pour écrire deux lignes à leurs frères ou amis... alors que beaucoup de ceux qui possèdent le génie de la langue et maîtrisent l'art de la prose et celui de la poésie seraient incapables de distinguer le sujet du complément et le nominatif du génitif ${ }^{2}$.»

Pour respecter les principes d'une pédagogie de succès et assurer aux étudiants une compétence communicative centrée sur une bonne utilisation de la pensée et de la langue, les cours de mise à niveau supposent des matériels et des stratégies spécifiques.

Des matériels pédagogiques publiés peuvent être utilisés ou du moins consultés. Mais il s'avère de plus en plus nécessaire de concevoir et d'élaborer des matériels répondant de manière adéquate aux objectifs de ces cours ${ }^{3}$. L'idéal reste, bien entendu, un matériel spécifique conçu à partir des besoins des étudiants mais jamais définitif, constamment ouvert aux innovations et évoluant dans une perspective foncièrement problématique et imperméable à toute tendance au figement ou au cloisonnement. Quoi qu'il en soit, le choix d'un matériel peut jouer un rôle décisif dans la marche d'un cours.

Mais il ne suffit pas de savoir choisir ou élaborer un matériel. Il s'agit surtout de réussir à l'utiliser et à l'exploiter dans le cadre d'une opération pédagogique motivante et épanouissante. L'enseignant veillera avant tout à jouer un rôle d'animateur. En mettant à la disposition des étudiants des documents variés, en proposant des exercices adéquats et en suscitant par les discussions une ambiance de communication interactive, il se comportera comme metteur en scène incitant les étudiants à jouer un rôle d'acteur et non de simple auditeur.

Cependant, tant que l'étudiant n'est pas engagé dans son propre apprentissage, le cours de mise à niveau restera une activité provisoire, voire inutile. L'enseignant aidera donc l'apprenant à individualiser sa mise à niveau et à acquérir les mécanismes de l'auto-évaluation. La production de l'étudiant, orale ou écrite, sera le point de départ de la plupart des activités proposées. L'auto-correction des travaux, suite aux remarques et notations de l'enseignant, reste le meilleur moyen de se rattraper et de progresser.

Étant individualisée, la mise à niveau ne saurait se contenter d'un nombre déterminé d'heures hebdomadaires. L'étudiant a besoin d'un environnement socio-culturel facilitant son perfectionnement. Il doit avoir les moyens de lire, d'écouter, de parler et d'écrire en dehors des cours. L'apprentissage d'une langue est une gymnastique individuelle et lente. C'est un comportement à acquérir, et non un programme à étudier et à assimiler en quelques semaines, voire en quelques mois. L'enseignant veillera à enrichir cet environnement et à faciliter cette gymnastique.

Dans cette perspective, la mise à niveau devient une pratique personnelle qui dépasse les différentes étapes de la formation et accompagne le traducteur et l'interprète tout au long de l'exercice de leur métier. Appelés à comprendre et à faire comprendre, 
ils se comportent continuellement comme lecteurs et producteurs privilégiés dans leurs différentes langues de travail.

\section{Conclusion}

Généralement conçue pour la deuxième et la troisième langues, la mise à niveau des étudiants arabophones s'étend nécessairement à la première langue, à cause des spécificités socio-linguistiques de l'arabe littéraire. Elle gagnerait à s'organiser en sessions intensives au début de la première année du cycle d'études aboutissant à la Licence en langues vivantes (mention traduction) préparatoire au Diplôme de Traducteur ou au Diplôme d'Interprète. Mais quelles que soient les formules proposées, cette mise à niveau demeure préalable à l'apprentissage de la traduction et de l'interprétation. En effet, si, en traduisant, le traducteur est invité à devenir co-écrivain ou écrivain à part entière, il reste qu'une acquisition solide des langues précède nécessairement l'opération traduisante et que ce n'est pas en traduisant qu'on peut apprendre une langue étrangère.

\section{NOTES}

1. Cet article s'appuie sur l'expérience de l'École de Traducteurs et d'Interprètes de Beyrouth de l'Université Saint-Joseph (fondée en 1980) qui recrute suite à un concours des candidats titulaires du Baccalauréat. Elle propose un cycle d'études de trois ans commun aux candidats à la traduction ou à l'interprétation et aboutissant à une Licence en langues vivantes (mention traduction). Après la licence, les options se précisent, soit pour le Diplôme de Traducteur, soit pour le Diplôme d'Interprète (deux ans d'études au minimum après la Licence).

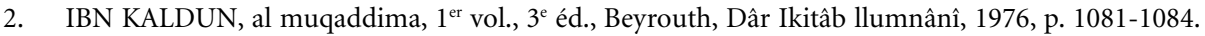

3. À titre d'exemple, l'Institut de Langues et de Traduction de l'Université Saint-Joseph a déjà élaboré deux matériels de mise à niveau susceptibles d'être utilisés à l'École de Traducteurs et d'Interprètes de Beyrouth:

- Qifâ Nahki pour l'arabe, couvrant 150 heures de cours (version définitive 1999);

- Expression-Communication pour le français, couvrant 100 heures de cours (version définitive 1999).

Un matériel d'anglais est en cours de préparation, version définitive prévue pour l'an 2000.

\section{RÉFÉRENCES}

Awaiss, H. (1997-1998): «Évaluation: Décider ensemble», Annales, Beyrouth, Institut de Langues et de Traduction, ${ }^{\circ}$ 4, 1997-1998.

Besse, H. (1985) : Méthodes et Pratiques des manuels de langue, Paris, Didier, CREDIF.

Buzan, T. (1984): Une tête bien faite, Paris, Éditions D’organisation.

Dejean Le FÉAL, K. (1976): «Le perfectionnement linguistique», Études de linguistique appliquée, $\mathrm{n}^{\circ} 24$, Paris, Didier.

De La Garanderie, A. (1980): Profils pédagogiques, Paris, Le Centurion.

Fray ha, A. (1980): fi llugati lûarabiyyati wa baûdi muskilâtihâ («À propos de la langue arabe et de quelques problèmes qu'elle pose»), Beyrouth, Dar Annahâr.

НовеiкA, M. (1997-1998): «Expression et communication en arabe», Annales, Beyrouth, Institut de langues et de traduction, $\mathrm{n}^{\circ} 4$.

Kfouri Khoury, N. (1998) : Prendre des notes vite et bien. Guide de l'enseignant, Paris, EDIFRAFrance.

Martinet, A. (1967): Éléments de linguistique générale, Paris, Armand Colin. 